\title{
A challenging case of tracheal foreign body retrieval following COVID-19 swabbing
}

\author{
James May', Katrina Mason², Parag Patel², Brendan Madden ${ }^{3}$ \\ ${ }^{1}$ Department of Respiratory Medicine; ${ }^{2}$ Department of ENT Surgery; ${ }^{3}$ Department of Cardiothoracic Medicine, St George's \\ University Hospital NHS Trust, London, UK
}

\begin{abstract}
The COVID-19 pandemic has resulted in a significant increase in the number of tracheostomised patients in hospitals requiring ventilatory support. These patients require highly specialist care, but overwhelmed hospital systems with stretched human resources potentially leave these patients cared for by undertrained healthcare professionals. We describe a rare complication where a routine COVID-19 swab done incorrectly via a tracheostomy tube, resulted in a snapped-off swab in the trachea. We outline the events and our method of removal using rigid bronchoscopy through the tracheostomy stoma as endo-tracheal bronchoscopy was impossible due to significant sub-glottic stenosis. This case highlights the paramount importance of the unique care needed to safely manage tracheostomies during this ongoing pandemic.
\end{abstract}

\section{Introduction}

Since emerging in the early part of 2020 , there have now been over 175 million cases of Coronavirus Disease 2019 (COVID-19)

Correspondence: Professor Brendan Madden, Department of Cardiothoracic Medicine, St George's University Hospital NHS Trust, London SW17 0QT, UK.

Tel. +44.020.87251094 - Fax: +44.020 .87252588 .

E-mail: brendan.madden@stgeorges.nhs.uk

Key words COVID-19; tracheostomy; foreign bodies; trachea; bronchoscopy.

Received for publication: 14 July 2021.

Accepted for publication: 22 September 2021.

Publisher's note: All claims expressed in this article are solely those of the authors and do not necessarily represent those of their affiliated organizations, or those of the publisher, the editors and the reviewers. Any product that may be evaluated in this article or claim that may be made by its manufacturer is not guaranteed or endorsed by the publisher.

${ }^{\circ}$ Copyright: the Author(s), 2021

Licensee PAGEPress, Italy

Monaldi Archives for Chest Disease 2022; 92:2014

doi: 10.4081/monaldi.2021.2014

This article is distributed under the terms of the Creative Commons Attribution-NonCommercial International License (CC BY-NC 4.0) which permits any noncommercial use, distribution, and reproduction in any medium, provided the original author(s) and source are credited. caused by the SARS-CoV-2 virus worldwide [1]. In the UK, over half a million people infected with the virus have been admitted to hospital [2] to manage the severe respiratory illness that can be caused by the infection. The NHS has had to rapidly adapt in response to the pandemic which has involved: creating double the number of pre-pandemic intensive care unit (ICU) beds; redeploying staff to undertake less familiar job roles; and requiring nurse to patient ratios to increase.

There have been high numbers of patients needing tracheostomies for both the short and longer term management of the illness. Traditionally, these patients have been cared for in specified areas of the hospital with appropriate trained staff. However, the adjustments of the healthcare system have meant the model of care of tracheostomies prior to the pandemic is now not always possible.

Our South London Trust now regularly tests all inpatients, including those with tracheostomies, for COVID-19. We use the Virocult ${ }^{\circledR}$ swab manufactured by Medical Wire \& Equipment Ltd. (Corsham, UK) to test patients using the polymerase chain reaction (PCR) method. This swab is designed for sampling from the nose and throat, with the plastic swab including a break point enabling the tip to be snapped-off and contained with the culture medium for transport to the lab.

We describe a complication whereby a patient with a tracheostomy was routinely tested for the virus incorrectly via the tracheostomy tube resulting in the swab breaking at the breakpoint and falling into the airway. Although this has been described previously [3] we encountered challenges in retrieving the foreign body and took a unique approach to successfully do so.

\section{Case Report}

A 35-year-old female patient with multiple co-morbidities was admitted to the intensive care unit (ICU) and ultimately required intubation and tracheostomy formation for ventilatory wean. Several failed extubation attempts demanded an inpatient assessment of the airway under anesthetic. Microlaryncoscopy and rigid bronchoscopy identified sub-glottic stenosis from granulation and scar tissue which was excised at the time of surgery.

She was stepped down from ICU to a specialist ear, nose, and throat (ENT) ward after 120 days with a focus on rehabilitation due to significant deconditioning. Due to further failed de-cannulation attempts, discharge with a long-term tracheostomy was planned, with further airway procedures to be considered when fully recovered as an out-patient. 
At this point in time, the trust had been divided into "COVID positive", "COVID suspected", and "COVID negative" wards. The ENT ward was designated a COVID negative ward and asymptomatic patients were routinely tested for the virus every 35 days.

Nine days after step-down, the patient was routinely swabbed for the virus by a registered nurse. For unclear reasons, the swab was done via the tracheostomy inner cannula and not via the nose or throat. This was not established procedure within our trust. As the swab was inserted in to the tracheostomy it snapped at the break point, with the tip falling through the inner tube. It was not visible on immediate inspection. This caused the patient to cough with episodic desaturation. High flow oxygen was administered as well as nebulized adrenaline.

Flexible nasendoscopy (FNE) through the tracheostomy was performed promptly by an ENT advanced nurse practitioner. The swab tip was visualized approximately $5 \mathrm{~mm}$ below the distal end of the tracheostomy tube (Figure 1).

The patient was taken to theatre and under general anaesthetic a flexible bronchoscope with grabbing forceps was introduced via the tracheostomy. The swab was seen once again but attempts to remove it failed and resulted in worsening airway oedema as reported by the ENT team. The procedure was abandoned and further nebulized adrenaline and intravenous corticosteroids were administered. A chest radiograph was taken that evening and there was no evidence of pneumothorax or lobar collapse and the swab tip - as expected - was not visible (Figure 2).

The patient was subsequently referred to our team which has experience in complex large airway intervention [4]. She underwent rigid and flexible bronchoscopy under a second general anaesthetic the following day. Due to significant subglottic stenosis the rigid bronchoscope was inserted directly via the tracheostomy stoma in the neck. The swab tip was visualized in the right main bronchus with the proximal end closely applied to the lateral tracheal wall. It was successfully removed with telescope forceps under direction visualisation. The retrieved tip measured approximately $5 \mathrm{~cm}$ (Figure 3 ). There was evidence of generalized airway oedema and associated oozing immediately following removal.

The patient returned to the ward and made a full recovery from the incident. Intravenous steroid was administered for a further 24 hours.

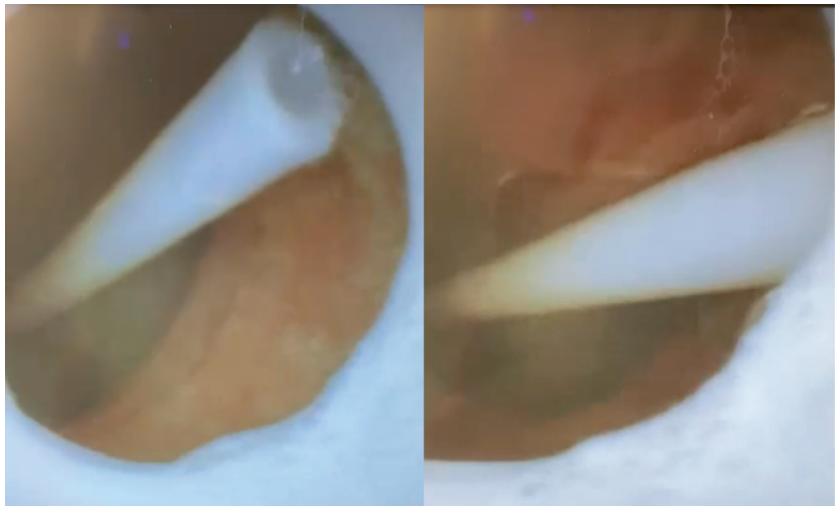

Figure 1. Flexible nasendoscopy performed via the tracheostomy inner tube showing the swab tip in the trachea. The distal end is not clearly visible.

\section{Discussion}

This case is similar to one described previously [3], however in this situation the patient was taken to theatre on two occasions as the position of the swab tip embedded in the lateral tracheal wall made it challenging to remove. We had to adopt a less conventional approach by inserting the rigid bronchoscope through the tracheal stoma as oral endo-tracheal intubation was not possible due to sig-

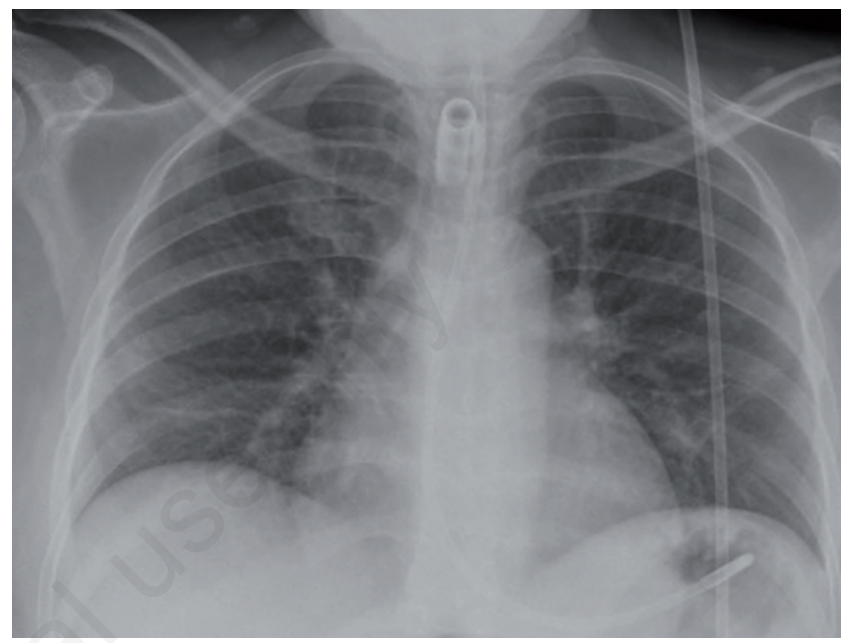

Figure 2. Anteroposterior chest radiograph. The tracheostomy is clearly seen but there is no evidence of radiopaque foreign body or other significant pathology.

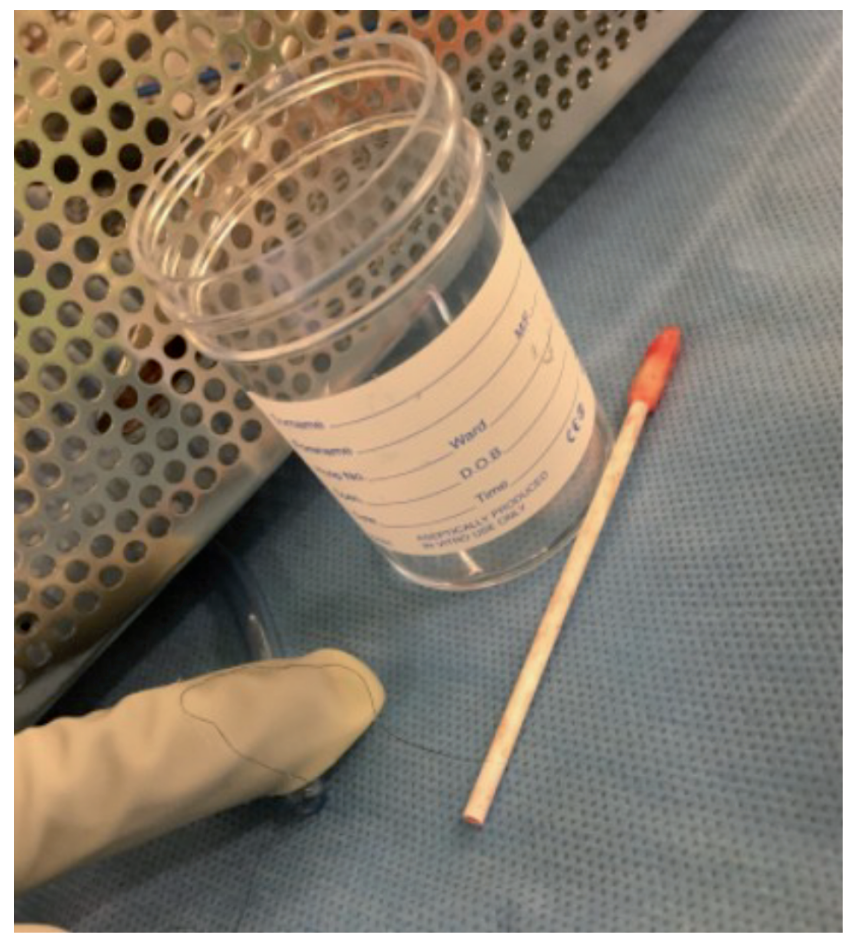

Figure 3. The bloodied tip retrieved intact from the main airway with telescope forceps. 
nificant sub-glottic stenosis. The used of rigid bronchoscopy conferred the already well-established benefit of allowing a greater degree of control for the operator as well as allowing for lung isolation in the event of major airway haemorrhage which was deemed to be a not insignificant risk.

Given that this is not the first time this complication has been reported, we were alarmed that such potential harm was possible with routine swabbing, especially as specialist airway intervention offered in only specialist centres - was necessary to retrieve the tip.

We therefore contacted the manufacturer and established that their position is that this swab should never be used in a tracheostomy for these reasons.

Our hospital guidelines at the time stated that samples should not "routinely" be taken from tracheostomies, however these have since been urgently revised to state the swab should "never" be used in tracheostomies. We appreciate that healthcare roles have had to adapt in response to the pandemic and we are certainly not placing blame or fault on the individuals involved in this case. In fact, we surveyed twenty members of the nursing staff who have been redeployed to work in ICU and 7 out of $20(35 \%)$ stated they were not in disagreement with the logic applied to swabbing a tracheostomy tube in preference to the nose/throat. However, all of those surveyed did say that they followed our trust policy of not using or caring for a tracheostomy unless they had the appropriate training to do so, unless in an absolute emergency.

With the possibility of a "third wave" on the horizon, as well as significant number of recovering patients with long term tracheostomies, the special attention needed for safe care of such patients needs re-emphasizing.

It may be that swab manufacturers look at reinforcing the safety warnings included with their product or that they adapt their swabs to be used safely with a tracheostomy to prevent the need for challenging intervention and potential harm.

\section{Summary and learning points}

- Patients with tracheostomies are likely to increase as result of the COVID019 pandemic placing a greater demand on the highly specialist care requisite of these patients.

- Unless there is an exceptional reason, testing for the virus should not be done through a tracheostomy and if it is felt appropriate should only be done after seeking the advice of an airway specialist.

- Rigid bronchoscopy through a tracheal stoma is a possible approach in foreign body retrieval in select cases, especially in complex cases which have failed conventional approaches.

\section{References}

1. World Health Organization. Coronavirus Disease (COVID-19) Dashboard. Available from: https://covid19.who.int

2. GOV.UK. Coronavirus (COVID-19) in the UK. Available from: https://coronavirus.data.gov.uk/details/healthcare

3. Hussain MH, Siddiqui S, Mahmood S. Tracheal swab from front of neck airway for SARS-CoV-2; a bronchial foreign body. BMJ Case Rep 2020;13:e237787.

4. Sharma A, Barnes N, Madden BP. Rigid bronchoscopy safety and outcomes - A single centre retrospective analysis. Poster presented at BTS Winter Meeting 2020. 